IMPERIAL/TP/2018/TM/01

\title{
Non-minimal gravitational reheating during kination
}

\author{
Konstantinos Dimopoulos ${ }^{a}$ and Tommi Markkanen ${ }^{b}$ \\ ${ }^{a}$ Consortium for Fundamental Physics, Physics Department, Lancaster University, Lancaster \\ LA1 4YB, United Kingdom \\ ${ }^{b}$ Department of Physics, Imperial College London, \\ Blackett Laboratory, London, SW7 2AZ, United Kingdom \\ E-mail: konst.dimopoulos@lancaster.ac.uk, t.markkanen@imperial.ac.uk
}

\begin{abstract}
A new mechanism is presented which can reheat the Universe in non-oscillatory models of inflation, where the inflation period is followed by a period dominated by the kinetic density for the inflaton field (kination). The mechanism considers an auxiliary field non-minimally coupled to gravity. The auxiliary field is a spectator during inflation, rendered heavy by the non-minimal coupling to gravity. During kination however, the non-minimal coupling generates a tachyonic mass, which displaces the field, until its bare mass becomes important, leading to coherent oscillations. Then, the field decays into the radiation bath of the hot big bang. The model is generic and predictive, in that the resulting reheating temperature is a function only of the model parameters (masses and couplings) and not of initial conditions. It is shown that reheating can be very efficient also when considering only the Standard Model.
\end{abstract}




\section{Contents}

1 Introduction $\quad 1$

2 The model $\quad 3$

3 Condensate creation during kination $\quad 4$

4 Reheating from the kination condensate $\quad 6$

$\begin{array}{lll}4.1 & \text { Case A: decay after vacuum restoration } & 7\end{array}$

4.1.1 Vacuum restoration after the field reaches $\chi_{\text {min }} \quad 8$

4.1.2 Vacuum restoration before the field reaches $\chi_{\min } \quad 9$

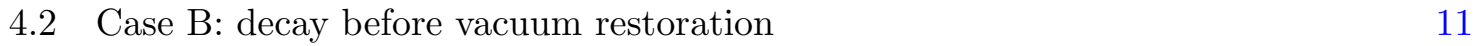

4.3 Relation to gravitational reheating 12

4.4 Concrete examples 13

$4.4 .1 \sqrt{\mathbf{6 \xi}}=\mathbf{1 2}(\boldsymbol{\xi}=\mathbf{2 4}) \quad 13$

$\begin{array}{lll}4.4 .2 \sqrt{\mathbf{6 \xi}}=\mathbf{1 0 0 0} & 14\end{array}$

5 Conclusions

\section{Introduction}

The most compelling mechanism for the generation of the primordial curvature perturbation which is responsible for the formation of structures in the Universe is the theory of Cosmic Inflation, which postulates that, in the beginning of its history, our Universe underwent a period of accelerated expansion. Inflation also accounts for the substantial fine-tuning of the initial conditions of Hot Big Bang cosmology, namely the infamous horizon and flatness problems. Observations of ever increasing precision are in definite support of the scenario of inflation. In particular, the observed primordial anisotropy in the Cosmic Microwave Background (CMB) radiation, suggests that the curvature perturbation is predominantly adiabatic and Gaussian and almost (but not quite) scale-invariant [1], in agreement with the simplest and most generic inflationary predictions.

In particle cosmology, inflation is realised through the inflationary paradigm, which considers that the Universe undergoes accelerated expansion when dominated by the potential density of a homogeneous scalar field. After the observation of the electroweak Higgs field, we are confident that fundamental scalar fields exist. One such field, called the inflaton, is assumed to control the dynamics of inflation. Recent CMB observations, from the Planck satellite, strongly suggest that the inflaton's scalar potential features a plateau with density roughly $10^{-10} M_{\mathrm{P}}^{4}[1,2]$. When the expectation value of the field is such that corresponds to the inflationary plateau, its potential density is roughly constant as the field slowly varies (rolls) along the plateau. At some point, the inflaton reaches the end of the plateau, where the scalar potential becomes steep and curved in field space, such that the field ceases to be potentially dominated and inflation ends. Traditionally, the inflationary paradigm suggests that the inflaton's scalar potential features a potential minimum not very far (in field space) from the inflationary plateau, such that, after inflation ends, the inflaton field oscillates 
around its vacuum expectation value (VEV) before decaying (perturbatively or not) into the thermal bath of the Hot Big Bang. The process is called (p)reheating.

However, in contrast to plateau inflation, which is supported by observations, the oscillatory reheating scenario described above is merely a conjecture. Indeed, many inflation models with a scalar potential that does not feature a minimum near the inflationary plateau have been considered over the years. Typically, the VEV of the inflaton field is displaced to infinity, such that, after the end of inflation, the field rolls down the steep runaway potential, being dominated by its kinetic density. This type of inflation is called non-oscillatory [3]. The period of kinetic density domination, which follows inflation, is called kination [4]. The attractive feature of such models is that the inflaton field avoids decay, which means that it can survive until today and play the role of quintessence (leading to late-time inflation) [5], which addresses the current dark energy observations that suggest that the Universe at present undergoes accelerated expansion again. Such quintessential inflation models [6] employ a single degree of freedom (the inflaton field) to provide a unified description of inflation and dark energy in the context of a common theoretical framework. However, non-oscillatory inflation can have other consequences as well, and does not need to be associated with dark energy. For example, a period of kination results in the number of inflationary e-folds corresponding to the cosmological scales exceeding the usual 60, which may have profound implications on the values of inflationary observables [7].

Since the inflaton field does not decay in non-oscillatory inflation models (as it does not have a particle interpretation) the formation of the thermal bath of the Hot Big Bang has to be achieved by other means. After reheating is somehow managed, the roll of the inflaton is halted and the field freezes at some value substantially displaced from the inflationary plateau, with some non-zero potential density, which can be the dark energy at present [6]. The most minimal approach to reheating in non-oscillatory inflation, which agrees with the economy of the quintessential inflation proposal, is the so-called gravitational reheating [8]. This is due to particle production of all light and conformally non-invariant fields during inflation, which generates a thermal bath characterised by the Hawking temperature of de Sitter space $T_{\mathrm{H}}=H / 2 \pi$, where $H$ is the inflationary Hubble scale. Gravitational reheating is inescapable but very inefficient. This means that kination lasts for a long time, before the generated radiation reheats the Universe and freezes the rolling field. Unfortunately, a prolonged kination period results in a large spike in the tensor power spectrum at small scales [9]. In gravitational reheating, this spike is large enough to challenge the process of Big Bang Nucleosynthesis (BBN), because the tensor density is almost $1 \%$ of the total during BBN. Thus, a reheating mechanism more efficient that gravitational reheating is preferable.

A mechanism which has been employed to efficiently reheat the Universe during kination is instant preheating [10]. This removes a fraction of the inflaton's kinetic density, through particle production when the inflaton crosses an Enhanced Symmetry Point (ESP) on the way down along its runaway potential, such that the mass of another field coupled to the inflaton changes non-adiabaticaly. This can be a very efficient mechanism, which easily overcomes the problem of excessive tensors. However, it needs to presuppose the existence of a suitable ESP at the right place in the inflaton direction, which means that it is rather restrictive model-building-wise. This is why a third option for reheating the Universe in non-oscillatory inflationary models has been considered, namely curvaton reheating [11].

The curvaton reheating mechanism considers the presence of a suitable auxiliary scalar field, called the curvaton [12]. The field is a spectator during inflation. It is assumed light such that it undergoes particle production during inflation, and develops thereby stochasticaly an 
expectation value displaced from the minimum along the curvaton direction. After the end of inflation, because the field is originally light, it remains frozen until the expansion rate decreases enough for the field to become heavy and begin coherent oscillations around its VEV. The decay of the curvaton field generates the desired radiation bath of the Hot Big Bang. Now, in the original curvaton proposal [12], the curvaton field also generated the dominant contribution to the curvature perturbations (hence it's name). For the reheating purposes however, this is not necessary and the curvaton's contribution to the curvature perturbations can be negligible. ${ }^{1}$ This proposal has the merit that no interaction is required with the inflaton, which means that inflation model-building is liberated [14]. However, it suffers from some drawbacks. In particular, the requirement that the curvaton remains light during inflation is non-trivial and amounts to some fine-tuning, especially in supergravity theories, in which masses of order $H$ are expected for all scalar fields, including the inflaton ( $\eta$-problem) [15]. Also, the expectation value of the curvaton during inflation is a crucial unknown parameter, which determines the generated reheating temperature, but depends strongly on the total duration of inflation [16], that is unknowable because of the no hair theorem. This strongly undermines the predictability of the proposal.

In this paper we propose a new mechanism which reheats the Universe in a nonoscillatory inflation model. Our mechanism makes use of the tendency of light scalar fields to become excited by the expansion of space, which happens quite generically in most cosmological spacetimes [17]. It enjoys the advantages of instant preheating and curvaton reheating, in that it can be highly efficient but also avoids their disadvantages in terms of not having an impact on inflation model-building (as has instant preheating, which requires a suitable ESP) and not requiring the appropriate tuning of initial conditions (as with curvaton reheating, which needs to specify the expectation value of the curvaton field in inflation). We consider the presence of an auxiliary scalar field, which is a spectator during inflation, much like the curvaton in curvaton reheating. However, our field is non-minimally coupled to gravity, which is a generic expectation as setting the non-minimal coupling to zero is not stable with respect to radiative corrections [18]. For a related reheating model making use of the non-minimal coupling, see Ref. [19]. During inflation this coupling makes the field heavy and it settles in the potential minimum, in contrast to the curvaton proposal. After inflation and during kination the inflationary minimum becomes a maximum in the scalar potential of our auxiliary field, which displaces the field at a value depended only on its non-minimal coupling to gravity (and possibly its self-coupling). Eventually, the bare mass of our field becomes important and the field oscillates around its VEV. It then may decay into the thermal bath of the Hot Big Bang. Our mechanism is generic and predictive because the generated reheating temperature depends only on the value of our model parameters such as masses and couplings.

We consider natural units, where $c=\hbar=k_{B}=1$ and $M_{\mathrm{P}}^{2} \equiv(8 \pi G)^{-1}$, with $G$ being Newton's gravitational constant and $M_{\mathrm{P}}=2.43 \times 10^{18} \mathrm{GeV}$ being the reduced Planck mass. Our conventions are $(+,+,+)[20]$.

\section{The model}

In this work we consider a non-oscillatory model of inflation in which, after the end of inflation, the influence of the potential to the field dynamics practically disappears leading

\footnotetext{
${ }^{1}$ The curvaton may decay into the radiation bath before or after it dominates the Universe. It may even lead to a brief period of inflation after it dominates but before it decays into radiation [13].
} 
to a phase of kinetic density domination (kination), i.e. an epoch that is described with the cosmological equation of state $w=p_{\phi} / \rho_{\phi}=1$, where $p_{\phi}$ and $\rho_{\phi}$ are the pressure and energy density of the inflaton field respectively. Besides considering a kination epoch after inflation we leave the specifics of inflation unfixed.

In addition to the inflaton we assume the existence of an originally subdominant, nonminimally coupled and massive scalar field with quartic self-interactions

$$
S_{\chi}=-\int d^{4} x \sqrt{|g|}\left[\frac{1}{2}\left(\partial_{\mu} \chi\right)^{2}+\frac{1}{2} m^{2} \chi^{2}+\frac{1}{2} \xi R \chi^{2}+\frac{1}{4} \lambda \chi^{4}\right],
$$

where $\xi \geq 1$. Importantly, we assume inflation to occur at such a high scale that during much of the kination period the bare mass $m$ of field is negligible with respect to the background Hubble rate.

Furthermore, the $\chi$-sector is assumed, for all practical purposes, to be decoupled from the inflaton. It is however assumed to have sizeable couplings to the Standard Model (SM) degrees of freedom. Therefore, $\chi$ could for example be the length of the Higgs doublet, but in order to keep our analysis as general as possible, the $\chi$-field may also be a generic beyond-the-SM field, with sufficient couplings to the SM.

\section{Condensate creation during kination}

The potential for the $\chi$-field can be read from (2.1)

$$
V(\chi)=\frac{1}{2} m^{2} \chi^{2}+\frac{1}{2} \xi R \chi^{2}+\frac{1}{4} \lambda \chi^{4},
$$

where $R=3(1-3 w) H^{2}$ is the curvature scalar. As we mentioned in the introduction, after the inflationary epoch when $w=-1$, the inflaton enters a period of kination with the equation of state $w=1$. By using the Friedmann equations

$$
\left\{\begin{array}{cl}
3 H^{2} M_{\mathrm{P}}^{2} & =\rho_{\phi} \\
-\left(3 H^{2}+2 \dot{H}\right) M_{\mathrm{P}}^{2} & =p_{\phi}=w \rho_{\phi}
\end{array},\right.
$$

it is straightforward to investigate the scale factor, the Hubble rate and the scalar curvature for a given equation of state parameter $w$. We have

$$
\begin{array}{ll}
\text { Inflation : } & a \propto e^{H t}, H=\text { constant, } R=12 H^{2}, \\
\text { Kination : } & a \propto t^{1 / 3}, \quad H=1 / 3 t, \quad R=-6 H^{2} .
\end{array}
$$




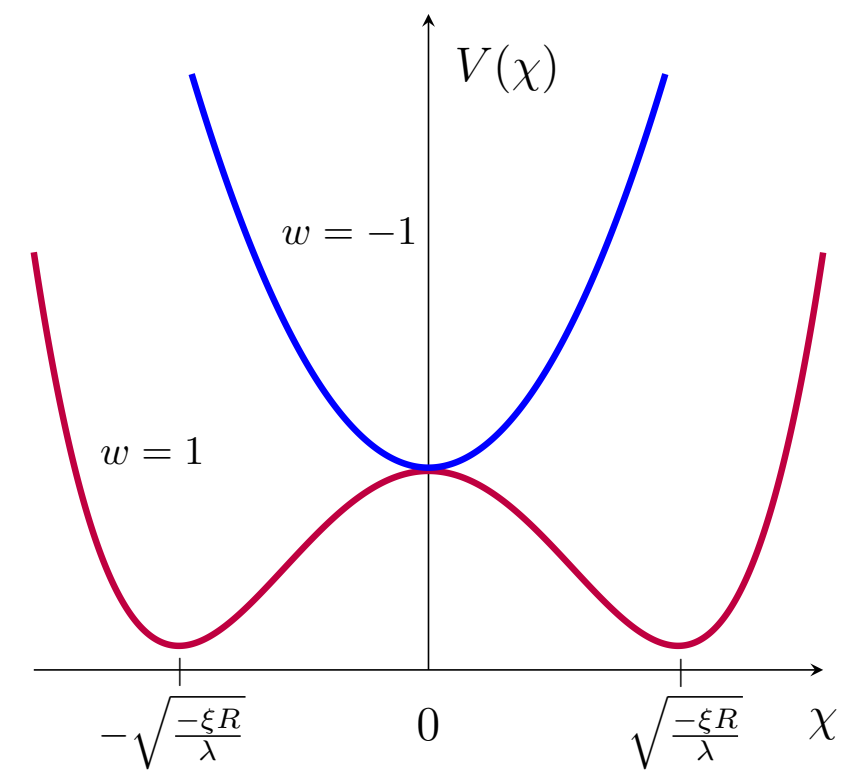

Figure 1. The potential (3.1) as a minimum at zero during inflation as described by the blue curve, but during kination if the tree-level mass parameter is smaller than $\sqrt{-\xi R}$ the potential will posses a minimum displaced from zero, as depicted by the purple curve.

Since we are assuming a positive non-minimal coupling with $\xi \geq 1$ and that the Hubble rate during inflation is much larger than the bare mass of the field, $H \gg m, \xi R$ gives the $\chi$ field a large positive mass parameter during inflation but becomes negative during kination. Therefore, the vacuum state is at zero during inflation

$$
V^{\prime}\left(\chi_{\min }\right)=0 \quad \Leftrightarrow \quad \chi_{\min }=0 ; \quad w=-1,
$$

but gets displaced from zero during kination as

$$
V^{\prime}\left(\chi_{\min }\right)=0 \quad \Leftrightarrow \quad \chi_{\min }^{2}=-\frac{\xi R+m^{2}}{\lambda} \approx-\frac{\xi R}{\lambda}=\frac{6 \xi}{\lambda} H^{2} ; \quad w=1,
$$

as depicted in Fig. 1. For now on, we refer to the non-zero value of the $\chi$-field in the minimum as the condensate. ${ }^{2}$

We may investigate the behaviour of the $\chi$-field by making use of a classical approximation i.e. study the behaviour of the zero mode. Since we are assuming that inflation takes place at a scale for which $m \ll H$ and that $\xi \geq 1$ giving $\chi=0$ during inflation, the

\footnotetext{
${ }^{2}$ The phase transition, which results in the creation of the non-zero condensate may also lead it the copious formation of topological defects. Such defects disappear when the vacuum at zero is restored, but they might influence the evolution of the Universe beforehand. The kind of defects produced, if any, and their stability depends on the actual theory considered. For example, if the $\chi$-field is complex then one may have the formation of cosmic strings, whose network, however, soon reaches a scaling solution and does not affect the Universe evolution. Similarly for higher order defects (textures). However, domain walls or monopoles would indeed affect the evolution of the Universe. Topological defects, even with transient existence, might lead to interesting effects but we do not investigate this possibility here. Instead, we assume that the evolution of the Universe is not affected by potential topological defects, so we can ignore them.
} 
equation of motion at the start of kination can be approximated by neglecting the mass and the quartic term in Eq. (2.1)

$$
\ddot{\chi}+3 H \dot{\chi}+\xi R \chi=0 \quad \Leftrightarrow \quad \ddot{\chi}+\frac{1}{t} \dot{\chi}-\frac{2 \xi}{3 t^{2}} \chi=0 .
$$

After inflation, the $\chi$-field becomes tachyonic. Thus, it is "kicked-off" the origin (which becomes a potential hill) due to quantum fluctuations, which are of typical amplitude $\delta \chi=$ $\left|m_{\text {eff }}\right| / 2 \pi$ per Hubble time $H^{-1},[17,22]$, where $m_{\text {eff }}$ is the tachyonic mass of the field, whose square is

$$
m_{\mathrm{eff}}^{2}=V^{\prime \prime}=m^{2}+\xi R \approx-6 \xi H^{2},
$$

where we ignored the bare mass of the $\chi$-field, which is $m \ll H$ near the end of inflation. Thus, as an initial condition we use $\chi_{\text {end }}=\sqrt{6 \xi} H_{\text {end }} / 2 \pi$ and $\dot{\chi}_{\text {end }}=H_{\text {end }} \chi_{\text {end }}$, with 'end' denoting the end of inflation. ${ }^{3}$

From the above we obtain

$$
\begin{aligned}
& \chi=\frac{H_{\text {end }}}{4 \pi}\left[(\sqrt{6 \xi}+1)\left(\frac{t}{t_{\text {end }}}\right)^{\sqrt{6 \xi / 3}}+(\sqrt{6 \xi}-1)\left(\frac{t}{t_{\text {end }}}\right)^{-\sqrt{6 \xi} / 3}\right] \Rightarrow \\
& \chi \simeq(\sqrt{6 \xi}+1) \frac{H_{\text {end }}}{4 \pi}\left[\left(\frac{t}{t_{\text {end }}}\right)^{1 / 3}\right]^{\sqrt{6 \xi}} \propto a^{\sqrt{6 \xi}} \text { when } t \gg t_{\text {end }} .
\end{aligned}
$$

Since $\chi_{\min } \propto a^{-3}$ the above indicates that the condensate quickly reaches the value $\sim \chi_{\min }$. We can estimate the Hubble rate when this occurs by comparing $\chi_{\min }$ in Eq. (3.6) to the above giving the condition

$$
\frac{\sqrt{\lambda}}{2 \pi}=\left(\frac{H_{m}}{H_{\text {end }}}\right)^{1+\sqrt{6 \xi} / 3}
$$

where the subscript ' $m$ ' denotes the moment when the $\chi$-field reaches the minimum of the potential $\chi=\chi_{\text {min }}$. Thus, we see that, considering a perturbative coupling $\lambda<4 \pi^{2}$, a regime always exists when the $\chi$-field is rolling down the central potential hill but has not reached the minimum of the potential yet, i.e. $H_{m}<H_{\text {end }}$ and $\chi<\chi_{\min }$.

\section{Reheating from the kination condensate}

For achieving successful reheating, the energy density of the $\chi$-field, $\rho_{\chi}$ (or its decay products), must start to dominate the evolution of the Universe over that of the inflaton $\rho_{\phi}$. It also must, before or after it has become dominant, decay into light SM degrees of freedom that thermalize. During kination we can see from Eqs. (3.2) and (3.4) that the energy-density of the inflaton scales as $\rho_{\phi} \propto a^{-6}$ and since from Eqs. (3.5) and (3.4) $\chi_{\min }^{2} \propto R \propto t^{-2} \propto a^{-6}$ we also see that in order for the energy density $\rho_{\chi}$ to overtake $\rho_{\phi}$ simply generating the condensate is not enough, but it or the energy density sourced by it must evolve to have a scaling less than $a^{-6}$.

In this work for completeness we assume a specific reheating model where the $\chi$-field is coupled to light fermions in the SM sector via a Yukawa-type interaction $\sim g \bar{\psi} \psi \chi$ giving rise to the decay rate

$$
\Gamma=g^{2} \frac{M}{8 \pi},
$$

\footnotetext{
${ }^{3}$ The value of $\chi_{\text {end }}$ would mildly change in the presence of more $\chi$-fields. As shown in Ref. [23], $\chi_{\text {end }}$ is proportional to $\sqrt{\mathcal{N}}$, where $\mathcal{N}$ is the number of fields. This would weakly affect our results but the effect is of order unity and can be ignored, at least for a number of fields which is not too large.
} 
where $g$ is a dimensionless coupling constant and $M$ the effective mass parameter for $\chi$. Note that this is not the tachyonic effective mass in Eq. (3.8) but it is given by $M=\sqrt{V^{\prime \prime}\left(\chi_{\min }\right)}$. From the potential in Eq. (3.1), when $\sqrt{6 \xi} H \gg m$ and $w=1$, we can obtain the mass as follows

$$
V^{\prime \prime}\left(\chi_{\min }\right)=m^{2}+\xi R+3 \lambda \chi_{\min }^{2}=2\left(6 \xi H^{2}-m^{2}\right) \simeq 12 \xi H^{2},
$$

with $\chi_{\min }=\sqrt{6 \xi / \lambda} H$. (cf. Eq. (3.6)). When $\sqrt{6 \xi} H \ll m$ we have $\chi_{\min }=0$ and so $M^{2}=m^{2}-6 \xi H^{2} \simeq m^{2}$, i.e. $M=m$. When $\Gamma<H$, the decay of $\chi$-particles into light fermions is exponentially suppressed. This means that the energy density stored in the $\chi$ field decays into the light fermions only when $\Gamma=H$. Actually, it is possible for the field not to decay even if $\Gamma>H$ if $\chi<\chi_{\min }$ and the field is still rolling down the potential hill. This is because, only after reaching the potential minimum can the field perform coherent oscillations and only an oscillating scalar field has a particle interpretation and can decay into fermions. ${ }^{4}$

In a model with a Yukawa interaction we can see that there are broadly two distinct scenarios in which the decay may happen. Soon after inflation when the kination condensate has been generated and we can still neglect the bare mass parameter $m$, by using $M=\sqrt{-2 \xi R}$ and Eq. (4.1) we obtain

$$
\frac{\Gamma}{H}=g^{2} \frac{\sqrt{3 \xi}}{4 \pi} .
$$

Thus, $\Gamma \geq H$ is possible when

$$
g^{2} \geq \frac{4 \pi}{\sqrt{3 \xi}} .
$$

When the above bound is satisfied the condensate practically decays immediately when the field reaches the minimum of the potential $\chi=\chi_{\min }$, i.e. at $H_{m}$ (as it cannot decay before). In contrast, when the above threshold is not satisfied, the $\chi$-field can decay only after $R$ has decreased such that the bare mass dominates $M$, the vacuum is restored at $\chi=0$ and one may approximate $M \approx m$ leading to a constant $\Gamma$, which eventually leads to the decay of the condensate.

Based on the above, in this work we consider two main scenarios for obtaining successful reheating as sourced by the condensate generated during kination:

A) The $\xi$ and $g$ do not satisfy the condition in Eq. (4.4). Thus, the condensate can decay only after the bare mass $m$ dominates over $\sqrt{-\xi R}$ and the vacuum is restored at $\chi=0$.

B) The condition in Eq. (4.4) is satisfied and the $\chi$-field decays into radiation immediately after reaching the minimum of the potential, which happens when $H=H_{m}$.

\subsection{Case A: decay after vacuum restoration}

If the constraint in Eq. (4.4) is not satisfied, the decay of the condensate does not take place near the end of inflation. After a sufficiently long time has passed, the scalar curvature decreases such that the bare mass term of the field can no longer be neglected. At this threshold, the minimum of the potential shifts back to $\chi=0$ restoring the vacuum configuration that was present prior to kination i.e. the blue curve in Fig. 1. Soon after this point,

\footnotetext{
${ }^{4}$ This decay of the scalar particles of the oscillating condensate should not be confused with the process of particle production, which creates particles due to the gravitational background during inflation. This process is discussed briefly in Sec. 4.3. Gravitational production of fermions is indeed possible [24], without the direct decay of the scalar condensate.
} 
the non-minimal and quartic terms become negligible and the field starts oscillating around $\chi=0$ in a quadratic potential with the energy density

$$
\rho_{\chi} \approx \frac{1}{2} m^{2} \chi^{2}
$$

which diluted as matter by the Universe expansion. We will approximate that immediately after the threshold

$$
m^{2}=-\xi R_{\mathrm{vr}}=6 \xi H_{\mathrm{vr}}^{2},
$$

where 'vr' stands for vacuum restoration, the density of the $\chi$-field starts scaling as $\rho_{\chi} \propto a^{-3}$. Indeed, the requirement that $H_{\mathrm{vr}}>\Gamma$, using $\Gamma=g^{2} m / 8 \pi$, results in the bound

$$
g^{2}<\frac{8 \pi}{\sqrt{6 \xi}}
$$

which is (roughly) the reverse of the bound in Eq. (4.4).

After reaching $H_{\mathrm{vr}}$ in Eq. (4.6), this scenario has two distinct ways of proceeding: vacuum restoration (meaning domination by the bare mass) can occur either before or after the field has reached the temporary minimum $\chi_{\text {min }}=\sqrt{-\xi R / \lambda}$, given in Eq. (3.6). In each case, the decay into radiation can occur either before or after the condensate has become the dominant component of the Universe content.

\subsubsection{Vacuum restoration after the field reaches $\chi_{\text {min }}$}

From the requirement $H_{m}>H_{\mathrm{vr}}$ it is straightforward to obtain

$$
\frac{\sqrt{\lambda}}{2 \pi}>\left(\sqrt{6 \xi} \frac{H_{\mathrm{end}}}{m}\right)^{-(1+\sqrt{6 \xi} / 3)},
$$

where we used Eqs. (3.10) and (4.6).

First we address the situation when the condensate decays into radiation before $\rho_{\phi}=\rho_{\chi}$. Using $\rho_{\phi} \propto a^{-6}$, the fact that the density of radiation scales as $\rho_{r} \propto a^{-4}$ and Eq. (4.6) we find that radiation starts dominating the evolution when

$$
\left.1 \equiv \frac{\rho_{\phi}}{\rho_{r}}\right|_{\mathrm{reh}}=\left.\left(\frac{a_{\mathrm{vr}}}{a_{\mathrm{dec}}}\right)^{3}\left(\frac{a_{\mathrm{dec}}}{a_{\mathrm{reh}}}\right)^{2} \frac{\rho_{\phi}}{\rho_{\chi}}\right|_{\mathrm{vr}}=\left(\frac{a_{\mathrm{vr}}}{a_{\mathrm{dec}}}\right)^{3}\left(\frac{a_{\mathrm{dec}}}{a_{\mathrm{reh}}}\right)^{2} \frac{\lambda M_{\mathrm{P}}^{2}}{\xi m^{2}},
$$

where 'dec' stands for decay and we considered that the condensate decays fully $\rho_{\chi}^{\text {dec }}=\rho_{r}^{\text {dec }}$. With the help of Eqs. (3.4) and (4.6), we straightforwardly get

$$
\left(\frac{a_{\mathrm{vr}}}{a_{\mathrm{dec}}}\right)^{3}=\frac{\Gamma}{H_{\mathrm{vr}}}=\frac{\sqrt{6 \xi} \Gamma}{m} ; \quad\left(\frac{a_{\mathrm{dec}}}{a_{\mathrm{reh}}}\right)^{2}=\left(\frac{H_{\mathrm{reh}}}{\Gamma}\right)^{2 / 3},
$$

allowing us to write from Eq. (4.9)

$$
H_{\mathrm{reh}}^{2 / 3}=\left(\frac{\xi}{6 \lambda^{2} \Gamma^{2 / 3}}\right)^{1 / 2} \frac{m^{3}}{M_{\mathrm{P}}^{2}} ; \quad \Gamma \geq H_{\mathrm{reh}} .
$$

The reheating temperature then comes straightforwardly using $3 H_{\mathrm{reh}}^{2} M_{\mathrm{P}}^{2}=\frac{\pi^{2}}{30} g_{*} T_{\text {reh }}^{4}$, which gives

$$
T_{\text {reh }}=\left(\frac{75}{2 \pi^{4}}\right)^{1 / 8}\left(\frac{\xi^{3 / 2}}{g_{*} \lambda^{3}}\right)^{1 / 4}\left(\frac{m^{9}}{M_{\mathrm{P}}^{4} \Gamma}\right)^{1 / 4}
$$


where $g_{*}$ is the number of effective relativistic degrees of freedom. Using $\Gamma=g^{2} m / 8 \pi$, the above can be writen as

$$
T_{\text {reh }}=\left(\frac{10}{3 \pi g_{*}}\right)^{1 / 4} \frac{1}{\sqrt{g}}\left(\frac{\sqrt{6 \xi}}{\lambda}\right)^{3 / 4} \frac{m^{2}}{M_{\mathrm{P}}} .
$$

Now, let us consider the situation when the field decays after $\rho_{\chi}=\rho_{\phi}$. In this case, we can easily write

$$
T_{\text {reh }}=\left(\frac{90}{\pi^{2} g_{*}} M_{\mathrm{P}}^{2} \Gamma^{2}\right)^{1 / 4},
$$

where we used Eq. (4.11) at the limit $\Gamma=H_{\text {dom }}$, with the 'dom' denoting the domination $\rho_{\phi}$ from $\rho_{\chi}$, i.e. $\rho_{\phi}^{\text {dom }}=\rho_{\chi}^{\text {dom }} . H_{\text {dom }}$ is straightforward to calculate, as follows

$$
\left.1 \equiv \frac{\rho_{\phi}}{\rho_{\chi}}\right|_{\mathrm{dom}}=\left.\frac{\rho_{\phi}}{\rho_{\chi}}\right|_{\mathrm{vr}}\left(\frac{a_{\mathrm{vr}}}{a_{\mathrm{dom}}}\right)^{3}=\frac{\lambda}{\xi}\left(\frac{M_{\mathrm{P}}}{m}\right)^{2} \frac{H_{\mathrm{dom}}}{H_{\mathrm{vr}}} \Rightarrow H_{\mathrm{dom}}=\frac{\sqrt{6 \xi}}{6 \lambda} \frac{m^{3}}{M_{\mathrm{P}}^{2}}
$$

where we used $\rho_{\phi}^{\mathrm{vr}}=3 H_{\mathrm{vr}}^{2} M_{\mathrm{P}}^{2}=\frac{3}{6 \xi}\left(m M_{\mathrm{P}}\right)^{2}$ and $\rho_{\chi}^{\mathrm{vr}}=\frac{1}{2} m^{2} \chi_{\mathrm{vr}}^{2}=m^{4} / 2 \lambda$, with $H_{\mathrm{vr}}=m / \sqrt{6 \xi}$ (cf. Eq. (4.6)) and $\chi_{\mathrm{vr}}=\sqrt{\frac{6 \xi}{\lambda}} H_{\mathrm{vr}}=m / \sqrt{\lambda}$ (cf. Eq. (3.6)). The requirement that $\Gamma<H_{\text {dom }}$ results in

$$
\Gamma<\left(\frac{\xi}{6 \lambda^{2}}\right)^{1 / 2} \frac{m^{3}}{M_{\mathrm{P}}^{2}} \Leftrightarrow g<\sqrt{\frac{4 \pi}{3 \lambda}}(\sqrt{6 \xi})^{1 / 2} \frac{m}{M_{\mathrm{P}}},
$$

where we used that $\Gamma=g^{2} m / 8 \pi$. Using this also in Eq. (4.14), we find

$$
T_{\mathrm{reh}}=\left(\frac{45}{32 g_{*}}\right)^{1 / 4} \frac{g}{\pi} \sqrt{m M_{\mathrm{P}}}
$$

The bounds in Eqs. (4.8) and (4.16) reinforce each other. Combining them together we get

$$
g<\frac{1}{\sqrt{3 \pi}}(\sqrt{6 \xi})^{\frac{3}{2}+\sqrt{6 \xi} / 3}\left(\frac{H_{\text {end }}}{m}\right)^{1+\sqrt{6 \xi} / 3} \frac{m}{M_{\mathrm{P}}} .
$$

\subsubsection{Vacuum restoration before the field reaches $\chi_{\text {min }}$}

From the requirement $H_{m}<H_{\mathrm{vr}}$ we obtain the reverse bound to the one in Eq. (4.8):

$$
\frac{\sqrt{\lambda}}{2 \pi}<\left(\sqrt{6 \xi} \frac{H_{\mathrm{end}}}{m}\right)^{-(1+\sqrt{6 \xi} / 3)},
$$

where we used Eqs. (3.10) and (4.6).

At vacuum restoration the value of the condensate is obtained as follows. Remember that, while the field is still rolling down the central potential hill, we have $\chi \propto a^{\sqrt{6 \xi}}$ (cf. Eq. (3.9)). This means that

$$
\chi_{\mathrm{vr}}=\chi_{\mathrm{end}}\left(\frac{a_{\mathrm{vr}}}{a_{\mathrm{end}}}\right)^{\sqrt{6 \xi}}=\frac{\sqrt{6 \xi} H_{\mathrm{end}}}{2 \pi}\left(\frac{H_{\mathrm{end}}}{H_{\mathrm{vr}}}\right)^{\sqrt{6 \xi} / 3},
$$

where $a \propto H^{-1 / 3}$ during kination. Using this, we obtain

$$
\left.\frac{\rho_{\phi}}{\rho_{\chi}}\right|_{\mathrm{vr}}=\frac{3 H_{\mathrm{vr}}^{2} M_{\mathrm{P}}^{2}}{\frac{1}{2} m^{2} \chi_{\mathrm{vr}}^{2}}=24 \pi^{2}(\sqrt{6 \xi})^{-4-\frac{2}{3} \sqrt{6 \xi}}\left(\frac{m}{H_{\mathrm{end}}}\right)^{2(1+\sqrt{6 \xi} / 3)}\left(\frac{M_{\mathrm{P}}}{m}\right)^{2},
$$




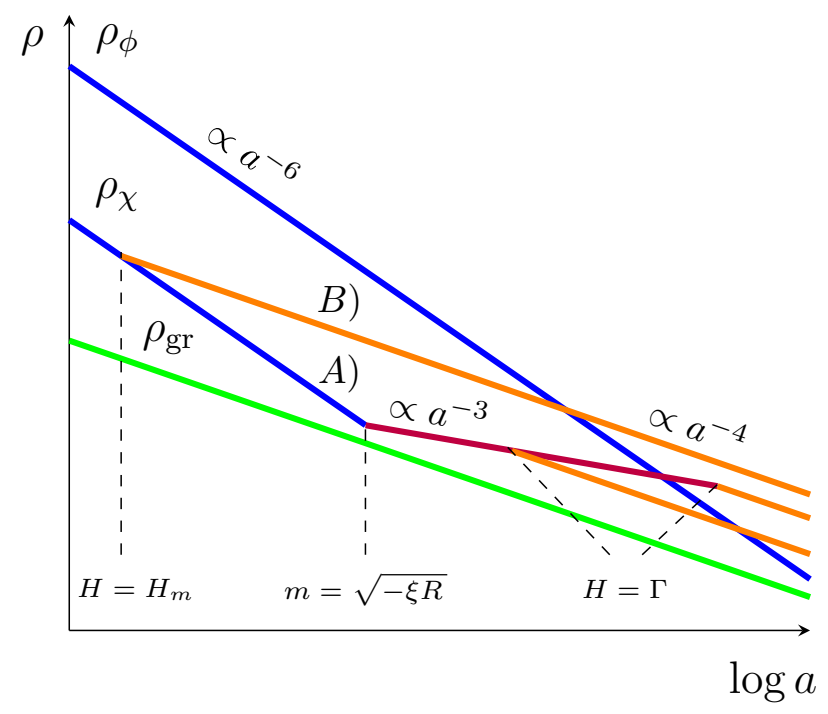

Figure 2. Illustration of two possible reheating mechanisms sourced by the condensate generated during kination, where the decay into SM particles is assumed to proceed through a Yukawa-coupling. The top blue curve labelled $\rho_{\phi}$ represents the dominant component of the energy density of the Universe i.e. the inflaton sector. The blue curve labelled $\rho_{\chi}$ is the energy-density of the $\chi$ sector, which can either A) decay into radiation $\propto a^{-4}$ (orange lines) after vacuum restoration containing a period of dust-like evolution $\propto a^{-3}$ (the red line) or B) decay before this happens. The green curve represents the energy density produced by gravitational reheating (see Sec. 4.3).

where we used that $H_{\mathrm{vr}}=m / \sqrt{6 \xi}$ (cf. Eq. (4.6)).

Consider first that the condensate decays after it dominates the Universe, meaning $\Gamma<H_{\text {dom }} . H_{\text {dom }}$ can be easily obtained as follows.

$$
\left.1 \equiv \frac{\rho_{\phi}}{\rho_{\chi}}\right|_{\mathrm{dom}}=\left.\frac{\rho_{\phi}}{\rho_{\chi}}\right|_{\mathrm{vr}}\left(\frac{a_{\mathrm{vr}}}{a_{\mathrm{dom}}}\right)^{3}=\left.\frac{\rho_{\phi}}{\rho_{\chi}}\right|_{\mathrm{vr}} \frac{H_{\mathrm{dom}}}{H_{\mathrm{vr}}},
$$

where we used $\rho_{\phi} / \rho_{\chi} \propto a^{-3}$ and $a \propto H^{-1 / 3}$ during kination. Thus, considering also Eq. (4.6), we get

$$
H_{\mathrm{dom}}=\frac{1}{24 \pi^{2}}(\sqrt{6 \xi})^{3+\frac{2}{3} \sqrt{6 \xi}}\left(\frac{H_{\mathrm{end}}}{m}\right)^{2(1+\sqrt{6 \xi} / 3)} \frac{m^{3}}{M_{\mathrm{P}}^{2}} .
$$

Demanding $\Gamma<H_{\text {dom }}$ results in a bound on $g$ identical to Eq. (4.18).

The reheating temperature is again given by Eq. (4.14) or Eq. (4.17). Thus, we see that if reheating of the Universe is achieved by the decay of the condensate into radiation after it has become the energetically dominant component, it makes no difference whether or not it has reached the potential minimum $\chi_{\text {min }}$ before vacuum restoration.

Now, let us consider the case when the condensate decays before domination. We find the reheating temperature as follows.

$$
\left.1 \equiv \frac{\rho_{\phi}}{\rho_{r}}\right|_{\mathrm{reh}}=\left.\frac{\rho_{\phi}}{\rho_{\chi}}\right|_{\mathrm{vr}}\left(\frac{a_{\mathrm{vr}}}{a_{\mathrm{dec}}}\right)^{3}\left(\frac{a_{\mathrm{dec}}}{a_{\mathrm{reh}}}\right)^{2}=\left.\frac{\rho_{\phi}}{\rho_{\chi}}\right|_{\mathrm{vr}} \frac{H_{\mathrm{dec}}}{H_{\mathrm{vr}}}\left(\frac{H_{\mathrm{reh}}}{H_{\mathrm{dec}}}\right)^{2 / 3},
$$

where $\rho_{\chi}^{\text {dec }}=\rho_{r}^{\text {dec }}$ and we considered that $\rho_{\phi} / \rho_{r} \propto a^{-2}$ and $\rho_{\phi} / \rho_{\chi} \propto a^{-3}$, with $a \propto H^{-1 / 3}$ during kination. Using Eq. (4.21) and considering that $H_{\mathrm{vr}}=m / \sqrt{6 \xi}$ (cf. Eq. (4.6)) and 
$H_{\mathrm{dec}}=\Gamma=g^{2} m / 8 \pi$, we calculate $H_{\mathrm{reh}}$ and subsequently obtain the reheating temperature, because $\frac{\pi^{2}}{30} g_{*} T_{\text {reh }}^{4}=3 H_{\text {reh }}^{2} M_{\mathrm{P}}^{2}$. Thus, we find

$$
T_{\text {reh }}=\left(\frac{5 \pi}{96 g_{*}}\right)^{1 / 4} \frac{1}{\pi^{2} \sqrt{g}}(\sqrt{6 \xi})^{\frac{1}{2}\left(\frac{9}{2}+\sqrt{6 \xi}\right)}\left(\frac{H_{\text {end }}}{m}\right)^{\frac{1}{2}(3+\sqrt{6 \xi})} \frac{m^{2}}{M_{\mathrm{P}}} .
$$

The condition $\Gamma>H_{\text {dom }}$ results in the bound

$$
g>\frac{1}{\sqrt{3 \pi}}(\sqrt{6 \xi})^{\frac{3}{2}+\sqrt{6 \xi} / 3}\left(\frac{H_{\mathrm{end}}}{m}\right)^{1+\sqrt{6 \xi} / 3} \frac{m}{M_{\mathrm{P}}},
$$

which is the reverse bound to Eq. (4.18).

\subsection{Case B: decay before vacuum restoration}

Now we investigate the behaviour of the condensate when Eq. (4.4) is satisfied and the condensated decays immediately after reaching $\chi_{\text {min }}$. Since the decay occurs before vacuum restoration we can neglect the bare mass parameter $m$ in the potential (3.1). Thus, the mass of the condensate when it reaches the potenital minimum $\chi_{\min }$ is given by Eq. (4.2) with $m=0, M=\sqrt{-2 \xi R}=2 \sqrt{3 \xi} H$. When the condensate reaches the potential miminum, its energy density can be estimated as

$$
\rho_{\chi}^{m} \approx \frac{1}{2} M^{2}\left(\chi_{\min }^{m}\right)^{2}=\frac{(6 \xi)^{2}}{\lambda} H_{m}^{4},
$$

where with ' $m$ ' we denote the moment that the condensate reaches the potential minimum $\chi=\chi_{\min }$ and we used that $\chi_{\min }^{m}=\sqrt{\frac{6 \xi}{\lambda}} H_{m}$ (cf. Eq. (3.6)). ${ }^{5}$ Thus,

$$
\left.\frac{\rho_{\phi}}{\rho_{\chi}}\right|_{m}=\frac{3 \lambda}{(6 \xi)^{2}}\left(\frac{M_{\mathrm{P}}}{H_{m}}\right)^{2},
$$

where we used $\rho_{\phi}^{m}=3 H_{m}^{2} M_{\mathrm{P}}^{2}$.

Reheating occurs at the moment when the radiation density overtakes that of the rolling inflaton field, which we can straightforwardly obtain analogously to the previous section by using Eq. (4.28) as

$$
\left.\left(\frac{a_{m}}{a_{\mathrm{reh}}}\right)^{2} \frac{\rho_{\phi}}{\rho_{\chi}}\right|_{m}=\left(\frac{H_{\mathrm{reh}}}{H_{m}}\right)^{2 / 3} \frac{3 \lambda M_{\mathrm{P}}^{2}}{(6 \xi)^{2} H_{m}^{2}}=1,
$$

leading to the reheating temperature

$$
T_{\text {reh }}=\left(\frac{90}{\pi^{2} g_{*}}\right)^{1 / 4}\left(\frac{6 \xi}{\sqrt{3 \lambda}}\right)^{3 / 2} \frac{H_{m}^{2}}{M_{\mathrm{P}}} .
$$

Using Eq. (3.10), the above can be recast as

$$
T_{\mathrm{reh}}=\left(\frac{90}{\pi^{2} g_{*}}\right)^{1 / 4}\left(\frac{6 \xi}{\sqrt{3 \lambda}}\right)^{3 / 2}\left(\frac{\sqrt{\lambda}}{2 \pi}\right)^{\frac{2}{1+\sqrt{6 \xi / 3}}} \frac{H_{\mathrm{end}}^{2}}{M_{\mathrm{P}}} .
$$

\footnotetext{
${ }^{5}$ We assumed that $\chi_{\min }^{m}>\chi_{\text {end }}$, which is equivalent to $\sqrt{\lambda} / 2 \pi<H_{m} / H_{\text {end }}$, which is true in view of Eq. (3.10) and $\xi>1$.
} 
The condensate should not dominate while it rolls down from the central potential hill. This is because, in such a case, the assumption of a background undergoing kination domination may not necessarily hold. This means we need $\rho_{\chi}^{m}<\rho_{\phi}^{m}$. In view of Eq. (4.27) and also Eq. (3.10) we find the bound

$$
\xi<\frac{\pi}{\sqrt{3}} \frac{M_{\mathrm{P}}}{H_{\mathrm{end}}}\left(\frac{\sqrt{\lambda}}{2 \pi}\right)^{\frac{1}{1+3 / \sqrt{6 \xi}}} .
$$

Case B is illustrated in Fig. 2 as the curve that starts out as orange.

\subsection{Relation to gravitational reheating}

Particle production via the generation of the kination condensate as described in this section is inherently a different mechanism to gravitational reheating, where quanta are produced from the transitional dynamics between the epoch of inflation and afterwards (e.g. a period of kination) [8]. Gravitational reheating results in general in a bath of relativistic particles and gravitational waves, whose density after its generation dilutes as $a^{-4}{ }^{6}$ We can estimate the energy density resulting from gravitational reheating at some time after the end of inflation as $[8]$

$$
\rho_{\mathrm{gr}}=q g_{*}^{\mathrm{gr}} \frac{\pi^{2}}{30}\left(\frac{H_{\mathrm{end}}}{2 \pi}\right)^{4}\left(\frac{a_{\mathrm{end}}}{a}\right)^{4},
$$

where $q \lesssim 1$ is an efficiency factor due to the fact that the density of radiation generated by gravitational reheating is of the order of thermal radiation with the Hawkingt emperature for de Sitter space $H_{\text {end }} /(2 \pi)$ but is not exactly thermal itself. The factor $g_{*}^{\text {gr }}$ includes all relativistic degrees of freedom produced by gravitational reheating, which are fields that are light (meaning with mass less than $H$ ) but not conformally invariant during inflation. Note that $g_{*}^{\mathrm{gr}}$ includes also the generated gravitational waves. The gravitational waves unavoidably generated from gravitational reheating can in some cases be boosted by an extended period of kination by such a large amount that obtaining successful Big Bang nucleosynthesis becomes non-trivial bounding the allowed parameter space [25].

In this work we are interested in parameter ranges for reheating from the kination condensate where the generated energy density is not overwhelmed by the one from gravitational reheating as illustrated by the green curve in Fig. 2. This leads to the condition

$$
\left.\frac{\rho_{\mathrm{gr}}}{\rho_{\chi}}\right|_{\mathrm{reh}}<1 \Leftrightarrow q g_{*}^{\mathrm{gr}} \frac{\pi^{2}}{30}\left(\frac{H_{\mathrm{end}}}{2 \pi}\right)^{4}\left(\frac{H_{\mathrm{reh}}}{H_{\mathrm{end}}}\right)^{4 / 3}<g_{*}^{\mathrm{reh}} \frac{\pi^{2}}{30} T_{\mathrm{reh}}^{4},
$$

where we have assumed that kination lasts until the moment of reheating. Using that $3 M_{\mathrm{P}}^{2} H_{\text {reh }}^{2}=g_{*}^{\text {reh } \frac{\pi^{2}}{30}} T_{\text {reh }}^{4}$ and after a little algebra, the above suggests

$$
T_{\text {reh }}>\frac{q^{3 / 4}}{24 \pi^{2}} \sqrt{\frac{g_{*}^{\text {gr }}}{10}}\left(\frac{g_{*}^{\text {gr }}}{g_{*}^{\text {reh }}}\right)^{1 / 4} \frac{H_{\mathrm{end}}^{2}}{M_{\mathrm{P}}} .
$$

Assuming $q \sim 1, g_{*}^{\mathrm{reh}} \approx g_{*}^{\mathrm{gr}}=\mathcal{O}(100)$ and inflation at the energy of grand unification we obtain the bound $T_{\text {reh }}>10^{5-6} \mathrm{GeV}$.

If the condensate decays after domination, we have a period after kination and before reheating that the Universe is dominated by the oscillating condensate, whose density scales

\footnotetext{
${ }^{6}$ Some particles produced gravitationally may become non-relativistic sometime after inflation, in which case their density scales as $a^{-3}$ and can become dark matter candidates [24].
} 
like matter and $a \propto H^{-2 / 3}$. Then, it is straightforward to show that the above lower bound on the reheating temperature is further relaxed by the factor $\Gamma / H_{\mathrm{dom}}$.

The precise relation of the reheating temperature to the model parameters depends on the specifics of the evolution. Namely, the reheating temperature to be put on the right-hand side in the above is given by (4.13), (4.17), (4.25) or (4.31).

Qualitatively, (4.34) leads to relevant bounds mostly for case (A) where the energy density of the condensate (potentially) has a lengthy period of $a^{-6}$ scaling, as is apparent from Fig. 2. Broadly speaking this will only be important for low reheating temperatures (4.35), which is also verified in the next section by going through the numerics of example cases.

\subsection{Concrete examples}

Here we will consider a few values for the non-minimal coupling $\xi$ and the other model parameters (the couplings $\lambda, g$ and the bare mass $m$ ) to demonstrate the efficiency of our reheating mechanism. We consider inflation at the scale of grand unification, as suggested by the CMB observations, with $H_{\text {end }} \sim 10^{-5} M_{\mathrm{P}} \sim 10^{13} \mathrm{GeV}$ and we take $g_{*}=106.75$, which corresponds to the standard model at high energies.

\subsection{1 $\sqrt{6 \xi}=12(\xi=24)$}

The bound in Eq. (4.7) becomes $g^{2}<2$, which is satisfied for a perturbative coupling. Therefore, the $\chi$-field condensate decays only after vacuum restoration, that is after the bare mass $m$ becomes important. Also, the bound in Eq, (4.8) becomes

$$
\frac{\sqrt{\lambda}}{2 \pi}>\left(\frac{m}{12 H_{\mathrm{end}}}\right)^{5}
$$

which turns up to be satisfied for most reasonable values of $m$ and $\lambda$ but not for very large $m$ (see below). This means that vacuum restoration occurs after the $\chi$-field reaches $\chi_{\text {min }}$. Now, Eq. (4.16) suggests

$$
\Gamma<H_{\mathrm{dom}} \Leftrightarrow g<4 \sqrt{\pi} \lambda^{-1 / 2} \frac{m}{M_{\mathrm{P}}} .
$$

If the above is satisfied, then the reheating temperature is given by Eq. (4.17), which becomes

$$
T_{\text {reh }} \sim 0.1 \times g \sqrt{m M_{\mathrm{P}}} .
$$

In contrast, if the bound in Eq. (4.37) is violated, then the reheating temperature is given by Eq. (4.13), which becomes

$$
T_{\text {reh }} \sim g^{-1 / 2} \lambda^{-3 / 4} \frac{m^{2}}{M_{\mathrm{P}}} .
$$

To see what the above imply, we select three indicative values for $m$ :

\section{$m \sim 1 \mathrm{TeV}$}

The bound in Eq. (4.36) becomes $\sqrt{\lambda}>10^{-54}$, which is well satisfied. The bound in Eq. (4.37) becomes $g<10^{-14} \lambda^{-1 / 2}$. If this is satisfied, $T_{\text {reh }}$ is given by Eq. (4.38), which is maximised for large $g$, which in turn is maximised for a small $\lambda$. We choose to consider $\lambda \sim 10^{-10}$. Then the bound on $g$ is $g<10^{-9}$. Choosing also $g \sim 10^{-10}$, Eq. (4.38) suggests that $T_{\text {reh }} \sim 1 \mathrm{GeV}$. 
Suppose now that the bound in Eq. (4.37) is violated, such that $g>10^{-14} \lambda^{-1 / 2}$. This means that $T_{\text {reh }}$ is now given by Eq. (4.39). Again $T_{\text {reh }}$ is maximised for small $\lambda$. Choosing again $\lambda \sim 10^{-10}$ we have $g>10^{-9}$. Choosing now $g \sim 10^{-8}$, Eq. (4.39) gives again $T_{\text {reh }} \sim 1 \mathrm{GeV}$. This is a rather small reheating temperature, which is overwhelmed by gravitational reheating as discussed in Sec. 4.3, especially when the condensate decays before it dominates the Universe.

\section{$m \sim 10^{10} \mathrm{GeV}$}

The bound in Eq. (4.36) becomes $\sqrt{\lambda}>10^{-19}$, which we expect again to be satisfied. The bound in Eq. (4.37) becomes $g<10^{-7} \lambda^{-1 / 2}$. If this is satisfied, $T_{\text {reh }}$ is given by Eq. (4.38), which is maximised for large $g$, which in turn is maximised for a small $\lambda$. We choose to consider again $\lambda \sim 10^{-10}$. Then the bound on $g$ is $g<10^{-2}$. Choosing also $g \sim 10^{-3}$, Eq. (4.38) suggests that $T_{\text {reh }} \sim 10^{10} \mathrm{GeV}$. Suppose now that the bound in Eq. (4.37) is violated, such that $g>10^{-7} \lambda^{-1 / 2}$. This means that $T_{\text {reh }}$ is now given by Eq. (4.39). Again $T_{\text {reh }}$ is maximised for small $\lambda$. Choosing again $\lambda \sim 10^{-10}$ we have $g>10^{-2}$. Choosing now $g \sim 0.1$, Eq. (4.39) gives again $T_{\text {reh }} \sim 10^{10} \mathrm{GeV}$. This is a sizeable value for $T_{\text {reh }}$, which demonstrates that reheating can be efficient in this model.

\section{$m \sim 10^{15} \mathrm{GeV}$}

The bound in Eq. (4.36) is badly violated. Instead, it is the bound in Eq. (4.19) that is satisfied. This means that vacuum restoration occurs before the $\chi$-field reaches $\chi_{\min }$. Now, Eq. (4.18) suggests

$$
\Gamma<H_{\text {dom }} \Leftrightarrow g<\left(\frac{12 H_{\text {end }}}{m}\right)^{5} \frac{m}{M_{\mathrm{P}}} .
$$

If the above is satisfied then $T_{\text {reh }}$ is again given by Eq. (4.38). If not, then $T_{\text {reh }}$ is determined by Eq. (4.25), which becomes

$$
T_{\text {reh }} \sim 0.1 \times g^{-1 / 2}\left(\frac{12 H_{\mathrm{end}}}{m}\right)^{15 / 2} \frac{m^{2}}{M_{\mathrm{P}}} .
$$

Inputting the value of $m$, the bound in Eq. (4.40) becomes $g<10^{-8}$. We choose $g \sim 10^{-9}$. Then Eq. (4.38) gives $T_{\text {reh }} \sim 10^{7} \mathrm{GeV}$. When the bound in Eq. (4.40) is violated and $g \geq 10^{-8}$, $T_{\text {reh }}$ is given by Eq. (4.41), which becomes $T_{\text {reh }} \sim g^{-1 / 2} 10^{3} \mathrm{GeV}$. Taking $g \sim 10^{-8}$, we obtain again $T_{\text {reh }} \sim 10^{7} \mathrm{GeV}$, which is the maximum value of $T_{\text {reh }}$ in this case.

\subsection{2 $\sqrt{6 \xi}=1000$}

We now consider a large value of $\xi$. The bound in Eq. (4.7) becomes $g<0.1$, which is possible to violate for a large but permissible value of $g$. In particular, the Yukawa coupling of the top quark does in fact violate this bound implying that this mechanism can be effective also when only the particle content of the Standard Model in considered. Let us consider this case first. Taking $g \sim 1$ we can satisfy the bound in Eq. (4.4). In this case the condensate decays immediately when it reaches the minimum of the potential at $\chi_{\min }$, i.e. at $H=H_{m}$, which is case B. The reheating temperature is now given by Eq. (4.31), which becomes

$$
T_{\mathrm{reh}} \sim 10^{8} \lambda^{-3 / 4} \frac{H_{\mathrm{end}}^{2}}{M_{\mathrm{P}}} \sim \lambda^{-3 / 4} \times 10^{16} \mathrm{GeV} .
$$


However, there is a lower bound on $\lambda$ coming from Eq. (4.32). Putting the numbers in, Eq. (4.32) suggests $\lambda \gtrsim 1$. Thus, $T_{\text {reh }} \sim 10^{16} \mathrm{GeV}$, which is the inflation scale and we have prompt reheating. This is no surprise as $H_{m}$ is reached right after the end of inflation. We also emphasize that the observed value for the Higgs four-point coupling $\lambda \sim 10^{-2}$ satisfies the bound Eq. (4.32) when the scale of inflation is lower by a few orders of magnitude from our chosen value of $10^{13} \mathrm{GeV}$, which implies that our mechanism can succesfully reheat the Universe via the Standard Model Higgs alone and can lead to high reheating temperatures $T_{\text {reh }} \sim 10^{12} \mathrm{GeV}$.

Consider now $g<0.1$, so that we are back in case A and the $\chi$-field condensate decays only after vacuum restoration, that is after the bare mass $m$ becomes important. The bound in Eq. (4.8) is overwhelmingly satisfied in all cases, so that vacuum restoration occurs after the $\chi$-field reaches $\chi_{\min }$. Eq. (4.16) suggests

$$
\Gamma<H_{\mathrm{dom}} \Leftrightarrow g<10^{3 / 2} \lambda^{-1 / 2} \frac{m}{M_{\mathrm{P}}} .
$$

If the above is satisfied, then the reheating temperature is given by Eq. (4.38). If the bound in Eq. (4.43) is violated, then the reheating temperature is given by Eq. (4.13), which now becomes

$$
T_{\text {reh }} \sim 10^{2} g^{-1 / 2} \lambda^{-3 / 4} \frac{m^{2}}{M_{\mathrm{P}}} .
$$

As before, to see what the above imply, we select the same three indicative values for $m$ :

\section{$m \sim 1 \mathrm{TeV}$}

The bound in Eq. (4.43) becomes $g<10^{-14} \lambda^{-1 / 2}$. If this is satisfied, $T_{\text {reh }}$ is given by Eq. (4.38), which is maximised for large $g$, which in turn is maximised for a small $\lambda$. We choose to consider $\lambda \sim 10^{-10}$. Then the bound on $g$ is $g<10^{-9}$. Choosing also $g \sim 10^{-10}$, Eq. (4.38) suggests that $T_{\text {reh }} \sim 1 \mathrm{GeV}$ as was always the case when $m \sim 1 \mathrm{TeV}$. Suppose now that the bound in Eq. (4.43) is violated, such that $g>10^{-14} \lambda^{-1 / 2}$. This means that $T_{\text {reh }}$ is now given by Eq. (4.44). Again $T_{\text {reh }}$ is maximised for small $\lambda$. Choosing $\lambda \sim 10^{-10}$ we have $g>10^{-9}$. Choosing now $g \sim 10^{-8}$, Eq. (4.44) gives again $T_{\text {reh }} \sim 10 \mathrm{GeV}$. These values of $T_{\text {reh }}$ are rather small. Such reheating temperature is overwhelmed by gravitational reheating (Sec. 4.3) especially when the condensate decays before it dominates the Universe.

\section{$m \sim 10^{10} \mathrm{GeV}$}

The bound in Eq. (4.43) becomes $g<10^{-7} \lambda^{-1 / 2}$. If this is satisfied, $T_{\text {reh }}$ is given by Eq. (4.38), which is maximised for large $g$, which in turn is maximised for a small $\lambda$. We choose to $\lambda \sim 10^{-10}$. Then the bound on $g$ is $g<10^{-2}$. Choosing also $g \sim 10^{-3}$, Eq. (4.38) suggests that $T_{\text {reh }} \sim 10^{10} \mathrm{GeV}$. Suppose now that the bound in Eq. (4.43) is violated, such that $g>10^{-7} \lambda^{-1 / 2}$. This means that $T_{\text {reh }}$ is now given by Eq. (4.44). Again $T_{\text {reh }}$ is maximised for small $\lambda$. Choosing again $\lambda \sim 10^{-10}$ we have $g>10^{-2}$. Selecting $g \sim 0.1$, Eq. (4.44) gives $T_{\text {reh }} \sim 10^{12} \mathrm{GeV}$. Note that the value $g \sim 0.1$ saturates the bound in Eq. (4.7).

$m \sim 10^{15} \mathrm{GeV}$

In contrast to the small $\xi$ case, when $\xi$ is large the bound in Eq. (4.8) is satisfied even for large values of $m$. Thus, the condensate decays only after vacuum restoration. The 
bound in Eq. (4.43) becomes now $g<10^{-2} \lambda^{-1 / 2}$. One does not need to consider very low values of $\lambda$. Choosing $\lambda \sim 10^{-2}$ we obtain $g<0.1$. This bound must be satisfied since a larger $g$ would violate the bound in Eq. (4.7). Then, the reheating temperature is given by Eq. (4.38), which suggests $T_{\text {reh }} \sim g \times 10^{16} \mathrm{GeV}$. Thus, saturating the upper bound on $g$ we obtain $T_{\text {reh }} \sim 10^{15} \mathrm{GeV}$.

Our results do not change dramatically when employing much bigger values of $\lambda$. For example, consider the case of $\xi=24$ and $m \sim 10^{10} \mathrm{GeV}$. The bound on $g$ is given by Eq. (4.37), which reads $g<10^{-7} \lambda^{-1 / 2}$. Suppose that we choose $\lambda \sim 10^{-4}$. Then following the same procedure as discussed above we find $T_{\text {reh }} \sim 10^{7} \mathrm{GeV}$ for $g \sim 10^{-5}$. If we choose $\lambda \sim 10^{-2}$, we obtain $T_{\text {reh }} \sim 10^{6} \mathrm{GeV}$ for $g \sim 10^{-7}$. It is more realistic to consider relatively large values of $\lambda$, which is the self-coupling of the $\chi$ scalar field, than $g$, which is a Yukawa coupling of $\chi$ to its fermionic decay products. We see that we may consider even $\lambda \sim 0.01$ at the expense of a few orders of magnitude on the value of $T_{\text {reh}}$, which is still decently large.

\section{Conclusions}

In this paper we studied a new mechanism to reheat the Universe during kination, which corresponds to an epoch dominated by the kinetic density of the inflaton field. Kination follows non-oscillatory inflation in which the inflaton field does not oscillate around its VEV but rolls down over large distances in field space. We have introduced a spectator field, non-minimally coupled to gravity, which can decay into the thermal bath of the hot big bang and terminate kination. Our spectator field is not coupled to the inflaton.

During inflation, the non-minimal coupling (assumed positive) renders the spectator field heavy and confines it at the origin. After the end of inflation and the onset of kination, however, the origin becomes a local maximum and the field is displaced towards the temporary minimum generated by the self-coupling of the field, which stabilises the scalar potential. Eventually, the bare mass of the field overwhelms the non-minimal term, the origin becomes again the minimum of the scalar potential (the VEV) and the spectator field coherently oscillates around its VEV until its decay into radiation, which reheats the Universe. We have investigated all possibilities, depending on the values of the bare mass and the couplings of the spectator field and showed that, with natural values of the model parameters, we can achieve successful reheating. For example, if the bare mass is around $m \sim 10^{10} \mathrm{GeV}$, the self-coupling is taken to be $\lambda \sim 0.01$ and the Yukawa coupling to light fermions is $g \sim 10^{-7}$ we obtain $T_{\text {reh }} \sim 10^{6} \mathrm{GeV}$. For large values of the bare mass one may even achieve prompt reheating, which occurs right after the end of inflation. For small values of $m$ with small $g$, however, the efficiency of reheating is not great. For example, when $m \sim 1 \mathrm{TeV}$, the typical value of the reheating temperature is $T_{\text {reh }} \sim 1 \mathrm{GeV}$, which is overwhelmed by gravitational reheating if our spectator field decays before dominating the Universe content.

In the context of Standard Model physics and other models with large Yukawa couplings, reheating can also be quite efficient with high reheating temperatures. As an example, we showed that if the Standard Model Higgs is non-minimally coupled to gravity with a nonminimal coupling $\sqrt{6 \xi} \sim 10^{3}$ and inflation ends at a scale $H_{\text {end }} \sim 10^{11} \mathrm{GeV}$ the Universe reheats with $T_{\text {reh }} \sim 10^{12} \mathrm{GeV}$.

We emphasise that our model is highly predictive and does not depend on initial conditions. In a variant of our model, it is easy to show that our spectator field may generate the observed dark matter if it decays into heavy fermions or not at all, provided that the Uni- 
verse is reheated by some other mechanism. A similar set-up for gravitational dark matter production was discovered in Ref. [26] and will be investigated in detail in Ref. [27].

When our paper appeared in arXiv another relevant paper where a similar set-up was studied [28] appeared simultaneously. In Ref. [28] however, the scalar field considered is the Higgs field, with only the interactions stipulated by the Standard Model. In our case, we consider the condensate dynamics in an extended framework encompassing also cases where the condensate does not decay immediately after inflation by allowing the mass, the four-point coupling as well as the Yukawa coupling to vary.

KD is supported (in part) by the Lancaster-Manchester-Sheffield Consortium for Fundamental Physics under STFC grant ST/L000520/1. TM is supported by the STFC grant $\mathrm{ST} / \mathrm{P} 000762 / 1$.

\section{References}

[1] P. A. R. Ade et al. [Planck Collaboration], Astron. Astrophys. 594 (2016) A13 [arXiv:1502.01589 [astro-ph.CO]]; N. Aghanim et al. [Planck Collaboration], arXiv:1608.02487 [astro-ph.CO].

[2] P. A. R. Ade et al. [BICEP2 and Keck Array Collaborations], Phys. Rev. Lett. 116 (2016) 031302 [arXiv:1510.09217 [astro-ph.CO]].

[3] G. N. Felder, L. Kofman and A. D. Linde, Phys. Rev. D 60 (1999) 103505 [hep-ph/9903350].

[4] B. Spokoiny, Phys. Lett. B 315 (1993) 40 [gr-qc/9306008]; M. Joyce and T. Prokopec, Phys. Rev. D 57 (1998) 6022 [hep-ph/9709320]; C. Pallis, JCAP 0510 (2005) 015 [hep-ph/0503080]; Nucl. Phys. B 751 (2006) 129 [hep-ph/0510234]; M. E. Gomez, S. Lola, C. Pallis and J. Rodriguez-Quintero, JCAP 0901 (2009) 027 [arXiv:0809.1859 [hep-ph]].

[5] B. Ratra and P. J. E. Peebles, Phys. Rev. D 37 (1988) 3406; P. J. E. Peebles and B. Ratra, Astrophys. J. 325 (1988) L17; K. Coble, S. Dodelson and J. A. Frieman, Phys. Rev. D 55 (1997) 1851 [astro-ph/9608122]; R. R. Caldwell, R. Dave and P. J. Steinhardt, Phys. Rev. Lett. 80 (1998) 1582 [astro-ph/9708069].

[6] R. A. Frewin and J. E. Lidsey, Int. J. Mod. Phys. D 2 (1993) 323 [astro-ph/9312035]; P. J. E. Peebles and A. Vilenkin, Phys. Rev. D 59 (1999) 063505 [astro-ph/9810509]; M. Peloso and F. Rosati, JHEP 9912 (1999) 026 [hep-ph/9908271]; S. C. C. Ng, N. J. Nunes and F. Rosati, Phys. Rev. D 64 (2001) 083510 [astro-ph/0107321]; K. Dimopoulos, Nucl. Phys. Proc. Suppl. 95 (2001) 70 [astro-ph/0012298]; G. Huey and J. E. Lidsey, Phys. Lett. B 514 (2001) 217 [astro-ph/0104006]; A. S. Majumdar, Phys. Rev. D 64 (2001) 083503 [astro-ph/0105518]; N. J. Nunes and E. J. Copeland, Phys. Rev. D 66 (2002) 043524 [astro-ph/0204115]; K. Dimopoulos, Phys. Rev. D 68 (2003) 123506 [astro-ph/0212264]; M. Sami and V. Sahni, Phys. Rev. D 70 (2004) 083513 [hep-th/0402086]; R. Rosenfeld and J. A. Frieman, JCAP 0509 (2005) 003 [astro-ph/0504191]; X. h. Zhai and Y. b. Zhao, Chin. Phys. 15 (2006) 2465 [astro-ph/0511512]; V. H. Cardenas, Phys. Rev. D 73 (2006) 103512 [gr-qc/0603013]; J. C. Bueno Sanchez and K. Dimopoulos, Phys. Lett. B 642 (2006) 294 Erratum: [Phys. Lett. B 647 (2007) 526] [hep-th/0605258]; J. C. Bueno Sanchez and K. Dimopoulos, JCAP 0710 (2007) 002 [hep-th/0606223]; A. Membiela and M. Bellini, Phys. Lett. B 641 (2006) 125 [gr-qc/0606119]; R. Rosenfeld and J. A. Frieman, Phys. Rev. D 75 (2007) 043513 [astro-ph/0611241]; I. P. Neupane, Class. Quant. Grav. 25 (2008) 125013 [arXiv:0706.2654 [hep-th]]; M. C. Bento, R. G. Felipe and N. M. C. Santos, Phys. Rev. D 77 (2008) 123512 [arXiv:0801.3450 [astro-ph]]; M. Bastero-Gil, A. Berera, B. M. Jackson and A. Taylor, Phys. Lett. B 678 (2009) 157 [arXiv:0905.2937 [hep-ph]]; M. C. Bento, R. Gonzalez Felipe and N. M. C. Santos, Int. J. Mod. Phys. A 24 (2009) 1639; M. Dias and A. R. Liddle, Phys. Rev. D 81 (2010) 083515 [arXiv:1002.3703 [astro-ph.CO]]; M. W. Hossain, R. Myrzakulov, M. Sami and E. N. Saridakis, Phys. Rev. D 90 (2014) no.2, 023512 
[arXiv:1402.6661 [gr-qc]]; Phys. Rev. D 89 (2014) no.12, 123513 [arXiv:1404.1445 [gr-qc]]; Phys. Lett. B 737 (2014) 191 [arXiv:1405.7491 [gr-qc]]; C. Q. Geng, M. W. Hossain, R. Myrzakulov, M. Sami and E. N. Saridakis, Phys. Rev. D 92 (2015) no.2, 023522 [arXiv:1502.03597 [gr-qc]]; A. R. Khalifeh, arXiv:1506.06250 [gr-qc]; J. Haro and S. Pan, arXiv:1512.03033 [gr-qc]; J. de Haro, Gen. Rel. Grav. 49 (2017) no.1, 6 [arXiv:1602.07138 [gr-qc]]; E. Guendelman, E. Nissimov and S. Pacheva, arXiv:1609.06915 [gr-qc]; J. Rubio and C. Wetterich, arXiv:1705.00552 [gr-qc]; C. Q. Geng, C. C. Lee, M. Sami, E. N. Saridakis and A. A. Starobinsky, arXiv:1705.01329 [gr-qc]; K. Dimopoulos and C. Owen, JCAP 1706 (2017) no.06, 027 [arXiv:1703.00305 [gr-qc]]; S. Ahmad, R. Myrzakulov and M. Sami, Phys. Rev. D 96 (2017) no.6, 063515 doi:10.1103/PhysRevD.96.063515 [arXiv:1705.02133 [gr-qc]]. A. Agarwal, R. Myrzakulov, M. Sami and N. K. Singh, Phys. Lett. B 770 (2017) 200 [arXiv:1708.00156 [gr-qc]]; K. Dimopoulos and C. Owen, arXiv:1712.01760 [astro-ph.CO].

[7] Y. Akrami, R. Kallosh, A. Linde and V. Vardanyan, arXiv:1712.09693 [hep-th].

[8] L. H. Ford, Phys. Rev. D 35 (1987) 2955; E. J. Chun, S. Scopel and I. Zaballa, JCAP 0907 (2009) 022 [arXiv:0904.0675 [hep-ph]].

[9] M. Giovannini, Class. Quant. Grav. 16 (1999) 2905 [hep-ph/9903263]; Phys. Rev. D 60 (1999) 123511 [astro-ph/9903004]; A. Riazuelo and J. P. Uzan, Phys. Rev. D 62 (2000) 083506 [astro-ph/0004156]; M. Yahiro, G. J. Mathews, K. Ichiki, T. Kajino and M. Orito, Phys. Rev. D 65 (2002) 063502 [astro-ph/0106349]; L. A. Boyle and A. Buonanno, Phys. Rev. D 78 (2008) 043531 [arXiv:0708.2279 [astro-ph]].

[10] G. N. Felder, L. Kofman and A. D. Linde, Phys. Rev. D 59 (1999) 123523 [hep-ph/9812289]; A. H. Campos, H. C. Reis and R. Rosenfeld, Phys. Lett. B 575 (2003) 151 [hep-ph/0210152].

[11] B. Feng and M. z. Li, Phys. Lett. B 564 (2003) 169 [hep-ph/0212213]; J. C. Bueno Sanchez and K. Dimopoulos, JCAP 0711 (2007) 007 [arXiv:0707.3967 [hep-ph]]; T. Matsuda, JCAP 0708 (2007) 003 [arXiv:0707.1948 [hep-ph]]; T. Qiu and K. Feng, Eur. Phys. J. C 77 (2017) no.10, 687 [arXiv:1608.01752 [hep-ph]].

[12] D. H. Lyth and D. Wands, Phys. Lett. B 524 (2002) 5 [hep-ph/0110002]; T. Moroi and T. Takahashi, Phys. Lett. B 522 (2001) 215 Erratum: [Phys. Lett. B 539 (2002) 303] [hep-ph/0110096]; K. Enqvist and M. S. Sloth, Nucl. Phys. B 626 (2002) 395 [hep-ph/0109214]; S. Mollerach, Phys. Rev. D 42 (1990) 313; A. D. Linde and V. F. Mukhanov, Phys. Rev. D 56 (1997) R535 [astro-ph/9610219].

[13] K. Dimopoulos, K. Kohri, D. H. Lyth and T. Matsuda, JCAP 1203 (2012) 022 [arXiv:1110.2951 [astro-ph.CO]].

[14] K. Dimopoulos and D. H. Lyth, Phys. Rev. D 69 (2004) 123509 [hep-ph/0209180].

[15] M. Dine, L. Randall and S. D. Thomas, Nucl. Phys. B 458 (1996) 291 [hep-ph/9507453]; Phys. Rev. Lett. 75 (1995) 398 [hep-ph/9503303].

[16] J. Torrado, C. T. Byrnes, R. J. Hardwick, V. Vennin and D. Wands, arXiv:1712.05364 [astro-ph.CO].

[17] T. Markkanen, JHEP 1801, 116 (2018) [arXiv:1711.07502 [gr-qc]].

[18] N. A. Chernikov and E. A. Tagirov, Ann. Inst. H. Poincare Phys. Theor. A 9 (1968) 109; C. G. Callan, Jr., S. R. Coleman and R. Jackiw, Annals Phys. 59 (1970) 42. E. A. Tagirov, Annals Phys. 76 (1973) 561.

[19] D. G. Figueroa and C. T. Byrnes, Phys. Lett. B 767 (2017) 272 [arXiv:1604.03905 [hep-ph]].

[20] C. W. Misner, K. S. Thorne and J. A. Wheeler, Gravitation, San Francisco 1973, W. H. Freeman and Company, p. 1279.

[21] G. N. Felder, J. Garcia-Bellido, P. B. Greene, L. Kofman, A. D. Linde and I. Tkachev, Phys. Rev. Lett. 87 (2001) 011601 [hep-ph/0012142]. 
[22] A. A. Starobinsky and J. Yokoyama, Phys. Rev. D 50, 6357 (1994) [astro-ph/9407016].

[23] I. F. Halpern, M. P. Hertzberg, M. A. Joss and E. I. Sfakianakis, Phys. Lett. B 748 (2015) 132 [arXiv:1410.1878 [astro-ph.CO]].

[24] D. J. H. Chung, L. L. Everett, H. Yoo and P. Zhou, Phys. Lett. B 712 (2012) 147 [arXiv:1109.2524 [astro-ph.CO]].

[25] M. L. Artymowski, O. Czerwinska, Z. Lalak and M. Lewicki, arXiv:1711.08473 [astro-ph.CO].

[26] T. Markkanen and S. Nurmi, JCAP 1702 (2017) 008 [arXiv:1512.07288 [astro-ph.CO]].

[27] M. Fairbairn, K. Kainulainen, T. Markkanen and S. Nurmi, Despicable Dark Matter, In preparation

[28] T. Nakama and J. Yokoyama, arXiv:1803.07111 [gr-qc]. 